\title{
GEOQUÍMICA E ÍNDICE DE GEOACUMULAÇÃO DE MERCÚRIO EM SEDIMENTOS DE SUPERFÍCIE DO ESTUÁRIO DE SANTOS - CUBATÃO (SP)
}

Wanilson Luiz-Silva*

Departamento de Geoquímica, Instituto de Química, Universidade Federal Fluminense, Outeiro São João Batista, s/n, 24020-007

Niterói - RJ

Rosa Helena R. Matos e Giane Chaves Kristosch

Departamento de Zoologia, Universidade Estadual Paulista, 13506-900 Rio Claro - SP

Recebido em 28/6/01; aceito em 9/11/01

\begin{abstract}
GEOCHEMISTRY AND INDEX OF GEOACCUMULATION OF MERCURY IN SURFACE SEDIMENTS FROM SANTOSCUBATÃO ESTUARY, STATE OF SÃO PAULO, BRAZIL. The concentrations of mercury in surface sediments $(<63 \mu \mathrm{m}$ fraction) from Santos-Cubatão Estuary, located in State of São Paulo, southeast Brazil, were determined to obtain the level of contamination in this highly polluted area. Fifteen stations distributed though seven rivers were sampled (156 samples collected) from September 1997 to August 1998 (12 stations sampled monthly) and during March, June, September and December 1998 (three stations sampled quarterly). The levels of mercury varied from 0.10 to $6.77 \mathrm{ppm}$, with the index of geoaccumulation of the rivers varying between grades 1 and 3 (annual average). The Cubatão is the most polluted river and the main agent of $\mathrm{Hg}$ dispersion in the study area. Geochemical data revealed that $\mathrm{Hg}$ levels in surface from Santos-Cubatão Estuary at present are as elevated as in past (for instance, by comparison 1980's), when Cubatão city was known as one of the most polluted cities in the world.
\end{abstract}

Keywords: heavy metal; mangrove; pollution.

\section{INTRODUÇÃO}

Os sedimentos, a água, o ar e a biota da área estuarina entre as cidades de Cubatão e Santos têm sido considerados contaminados especialmente por metais pesados, em alguns trabalhos ${ }^{1-4}$. A cidade de Cubatão abriga um importante pólo industrial do Brasil, contendo mais de 100 fábricas, que são as principais responsáveis pela origem da poluição ${ }^{5}$. As incontroláveis emissões de sólidos, líquidos e substâncias gasosas de indústrias químicas, petroquímicas, de fertilizantes, além de uma grande siderúrgica, colocaram a cidade de Cubatão, especialmente na década de 1980, no cenário das discussões ambientais mundiais. A poluição descontrolada conduziu Cubatão, naquela época, ao reconhecimento internacional de "uma das cidades mais poluídas do mundo". A partir de então, programas governamentais de controle da poluição foram aplicados na região, com o intuito de reverter este cenário caótico. O ecossistema aquático das áreas adjacentes ao setor industrial tem sofrido, além da ação das intensas descargas de efluentes industriais, despejos domésticos, os quais têm acarretado profundas transformações no meio ambiente ${ }^{1}$.

Apesar da poluição em grande escala na área da cidade de Cubatão, poucos trabalhos detalhados foram desenvolvidos para acompanhar a distribuição de metais pesados em sedimentos fluviais. Os trabalhos prévios de geoquímica em sedimentos foram desenvolvidos de 1974 a 1987, em períodos intermitentes e apenas em nível de reconhecimento, sendo realizados no interior da Baía de Santos e, com menor intensidade, na área do estuário, especialmente no Rio Cubatão ${ }^{1,2}$. Estes estudos fizeram parte dos programas governamentais de avaliação das condições ambientais e seus resultados denunciaram uma preocupante poluição de metais pesados na época em que foram realizados.

O presente trabalho apresenta dados da distribuição geoquímica de $\mathrm{Hg}$ em sedimentos superficiais de alguns rios do Estuário de San-

*e-mail: wanilsonls@bol.com.br
tos-Cubatão, com o objetivo de avaliar as condições atuais deste ambiente altamente impactado. Tais dados fazem parte de uma avaliação detalhada da distribuição de metais traços na área deste estuário (projeto em andamento). A amostragem teve início em setembro de 1997 e foi concluída em dezembro de 1998. Dados geoquímicos prévios da área em estudo ${ }^{1}$ são apresentados para comparação.

\section{PARTE EXPERIMENTAL}

\section{Materiais e métodos}

A área de estudo apresenta aproximadamente $30 \mathrm{~km}^{2}$ e cobre parte dos manguezais entre as cidades de Cubatão (situada à montante dos rios investigados) e Santos (à jusante), no Estado de São Paulo (Figura 1). Está localizada em uma estreita faixa ladeada pelo Oceano Atlântico e as escarpas da Serra do Mar. A área apresenta algumas dezenas de rios e canais de maré, e alguns são usados para despejos de águas industriais contaminadas.

Quinze estações, totalizando 156 amostras de sedimento, foram usadas neste estudo e distribuídas no Largo do Caneu (estações GD, GE e GF), que é um canal de fluxo de navios, e em alguns de seus tributários, como os rios Cubatão (estações GA, GB e GC), Cascalho (estações GG, GH e GI), Casqueiro (estações RA, RB e RC), Capivari (estação GL), Morrão (estação GJ) e Pedreira (estação GK) - Figura 1. Com exceção das estações GL, GJ e GK, que foram amostradas nos meses de março, junho, setembro e dezembro de 1998, as demais estações foram amostradas mensalmente durante o período de setembro de 1997 a agosto de 1998.

A amostragem em cada estação foi pontual, procurando-se amostrar sempre os mesmos locais. As amostras continham aproximadamente $0,5 \mathrm{~kg}$ de sedimento e foram coletadas em locais rasos (maré média) de sedimentação recente (sedimento ativo de corrente). A coleta foi realizada com as mãos, protegidas por luvas plásticas, e os sedimentos acondicionados em sacos plásticos devidamente etiquetados. Em laboratório, o material foi secado em estufas a 


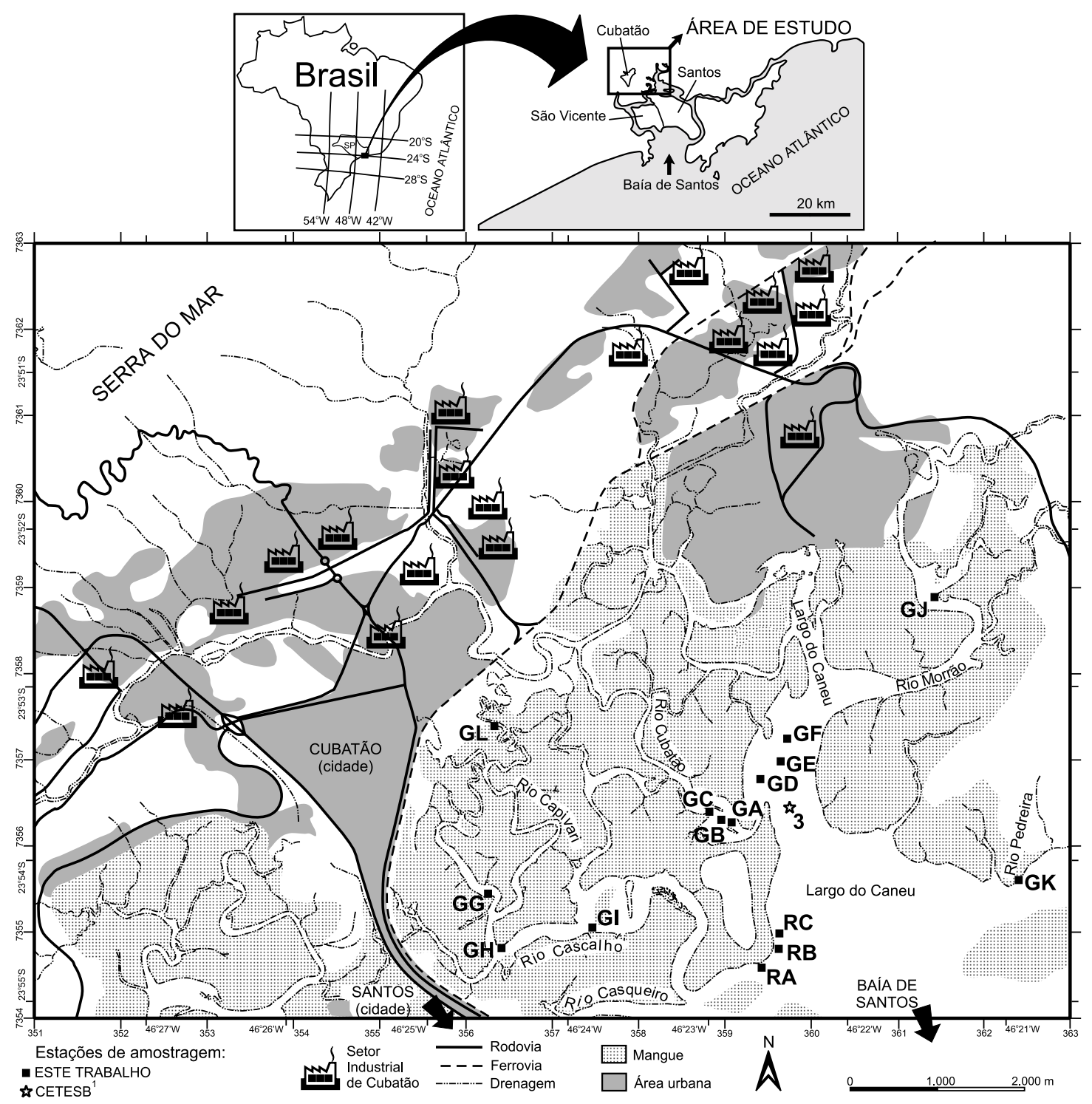

Figura 1. Mapa simplificado da área do Estuário de Santos-Cubatão (SP) com a localização das estações de amostragem

temperaturas de aproximadamente 25 a $30{ }^{\circ} \mathrm{C}$ e posteriormente, homogeneizado e quarteado manualmente. Cerca de $100 \mathrm{~g}$ de amostra foram então submetidas ao peneiramento por via seca para separar a fração silte-argila $(<63 \mu \mathrm{m})$ da areia $(>63 \mu \mathrm{m})$. Este tipo de peneiramento, comparativamente ao peneiramento por via úmida, é mais limpo e pode ser adequadamente usado na investigação do conteúdo de metais em sedimentos ${ }^{6}$. A fração $<63 \mu \mathrm{m}$ foi utilizada para medir a concentração de $\mathrm{Hg}$, tendo em vista que ela tende a concentrar mais os metais ${ }^{6-12}$.

As análises químicas foram realizadas no Laboratório Bondar Clegg (Vancouver, Canadá). Para o procedimento analítico, 0,5 g de amostra foi digerida em água régia, a uma temperatura de $90{ }^{\circ} \mathrm{C}$, durante 2,5 h. Após esfriar, o material foi diluído com solução de $\mathrm{HCl}$ fraco e a concentração do $\mathrm{Hg}$ determinada por vapor a frio, em um espectrômetro de absorção atômica (Varian AAS, modificado por Bondar Clegg). O limite de detecção instrumental foi de 10 ppb e os resultados foram certificados por dois padrões CCRMP (Canadian Certified Reference Materials Project; amostras denominadas LKSD-2 e STSD-4) da CANMET (Mining and Mineral
Sciences Laboratories). Amostras tipo branco e duplicadas também foram usadas para controle.

Para checar os graus de homogeneização química e granulométrica, quatro amostras foram divididas em duas porções, que foram peneiradas e analisadas. As diferenças na reprodução dos resultados foram inferiores a $2 \%$, para a granulometria, e entre $0,012 \mathrm{e}$ 0,186 ppm, para a concentração de $\mathrm{Hg}$. São diferenças relativamente pequenas, mesmo para o $\mathrm{Hg}$, tendo em vista as elevadas concentrações deste metal na área de estudo.

Para quantificar a poluição foi usado o "índice de geoacumulação" $\left(I_{g e o} ; \mathrm{cf}^{13}\right)$ :

$I_{\text {geo }}=\log _{2}\left[C_{n}\left(1,5 B_{n}\right)^{-1}\right]$

onde $C_{n}$ é a concentração medida do metal $n$ na fração fina do sedimento ( $<63 \mu \mathrm{m})$ e $B_{n}$ é o valor geoquímico de "background" desse metal baseado na composição média dos folhelhos $\left(\mathrm{Hg}=0,18 \mathrm{ppm}^{14}\right)$. O fator 1,5 da equação é usado para compensar possíveis variações dos dados de "background" devido a efeitos litogênicos ${ }^{13}$. O $I_{\text {geo }}$ é 
amplamente usado em trabalhos de avaliação geoquímica de ambientes impactados. A utilização da composição média dos folhelhos como referência ("background") global permite que o grau de contaminação de áreas diferentes possa ser comparado. O $I_{\text {geo }}$ consiste de sete graus: $0\left(I_{\text {geo }}<0\right), 1\left(I_{\text {geo }} \rightarrow>0 \mathrm{a} \leq 1\right), 2\left(I_{\text {geo }} \rightarrow>1 \mathrm{a} \leq 2\right), 3\left(I_{\text {geo }}\right.$ $\rightarrow>2 \mathrm{a} \leq 3), 4\left(I_{\text {geo }} \rightarrow>3 \mathrm{a} \leq 4\right), 5\left(I_{\text {geo }} \rightarrow>4 \mathrm{a} \leq 5\right)$ e $6\left(I_{\text {geo }}>5\right)$. O grau 6 reflete um enriquecimento de 100 vezes acima dos valores médios da composição dos folhelhos.

\section{RESULTADOS E DISCUSSÃO}

As distribuições granulométricas revelam diferenças significativas entre as amostras de uma mesma estação. Geralmente, a porcentagem em peso da fração $<63 \mu \mathrm{m}$ é menor do que a fração $>63 \mu \mathrm{m}$, predominando, portanto, sedimentos constituídos por areia fina com menor quantidade de silte-argila (média $=29,18 \pm 10,97 \%$ em peso). A variabilidade da granulometria dos sedimentos em uma mesma estação de amostragem é um testemunho da renovação constante do material.

Os resultados da geoquímica são mostrados resumidamente na Tabela 1 e Figura 2. A concentração absoluta de Hg ocorreu no intervalo de 0,10 a $6,77 \mathrm{ppm}$. As maiores concentrações ocorreram nos rios Cubatão (média anual $=1,73 \pm 0,94$ ppm), Largo do Caneu $(1,04 \pm 0,25 \mathrm{ppm})$ e Casqueiro (1,04 $\pm 0,40 \mathrm{ppm})$. Particularmente nestes rios, as concentrações mensais de $\mathrm{Hg}$ não revelam um padrão uniforme de distribuição (Figura 2). Cada rio, e até mesmo cada estação dentro do rio, mostra um padrão de variabilidade particular. Esta peculiaridade denuncia uma taxa de acumulação de $\mathrm{Hg}$ muito irregular. Os rios Pedreira, Morrão, Cascalho e Capivari, em ordem decrescente, apresentam os valores de concentração mais baixos, no intervalo de $0,31 \pm 0,11$ a $0,59 \pm 0,02 \mathrm{ppm}$. Nestes rios, a variabilidade mensal da concentração de $\mathrm{Hg}$ é menos significativa.

O $I_{\text {geo }}$ de $\mathrm{Hg}$ (média anual) coloca-se entre os graus 1 e 3, sendo que os maiores valores são encontrados no Rio Cubatão (grau 3), seguido pelos rios Casqueiro, Largo do Caneu e Pedreira, todos com grau 2. Na estação GC, a mais a montante do Rio Cubatão, o $I_{\text {geo }}$ de $\mathrm{Hg}$ (média anual) alcança o grau 4, com a concentração por amostra podendo superar em 37 vezes o valor de referência (grau 5). Os rios Cascalho, Morrão e Capivari apresentam os menores valores de $I_{g e o}$ de $\mathrm{Hg}$ (grau 1 = média anual).

O Rio Cubatão contém as maiores concentrações de $\mathrm{Hg}$ e constitui o principal veículo poluidor do estuário. Como as condições físico-químicas do ambiente estuarino naturalmente são propensas à precipitação dos metais (e.g. pH alcalino), em oposição à sua mobilidade (e.g. pH ácido), as concentrações de $\mathrm{Hg}$ nos sedimentos tendem a ser mais significativas nas proximidades da fonte poluidora, especialmente o setor industrial de Cubatão ${ }^{1,2}$. Isso é claramente mostrado pelas concentrações mais elevadas de $\mathrm{Hg}$ no Rio Cubatão, em relação aos demais rios investigados. Este rio recebe a principal carga de efluentes do setor industrial de Cubatão e lança suas águas poluídas no Largo do Caneu que, em virtude do movimento das marés, avança suas águas sobre as drenagens tributárias, promovendo a dispersão do metal. Esta dispersão é mais significativa nas áreas das drenagens próximas à desembocadura no Largo do Caneu, como mostram as concentrações relativamente elevadas de $\mathrm{Hg}$ nas estações de amostragem nos rios Pedreira e Casqueiro. Locais mais afastados, como as estações dos rios Capivari, Cascalho e Morrão, são menos afetados.

Dados antigos de concentração de $\mathrm{Hg}$ em sedimentos superficiais de uma estação de amostragem no Largo do Caneu (estação 3, amostragem em junho, setembro, dezembro/1979 e março/1980) ${ }^{1}$ podem ser usados em comparação com os atuais níveis de concentração. Esta estação localiza-se muito próxima às estações GD, GE e GF, amostradas neste trabalho (ver Figura 1), e ambos os resultados são praticamente idênticos (Tabela 1). Este fato denuncia que os níveis de concentração de $\mathrm{Hg}$ medidos em 1997/1998 nos sedimentos superficiais do Estuário de Santos-Cubatão mantêm-se semelhantes a aqueles dos anos 1979/1980, época na qual a problemática poluição de Cubatão veio à tona. Embora deva-se admitir que os poluentes presentes nos sedimentos não necessariamente constituem uma fon-

Tabela 1. Resumo dos resultados de concentração de $\mathrm{Hg}$ nos sedimentos superficiais (fração <63 $\mu \mathrm{m}$ ) do Estuário de Santos-Cubatão (SP). As estações GL, GK, GJ e estação 3 apresentam quatro amostras e as demais estações, 12 amostras. A média de Hg nos folhelhos é mostrada para comparação

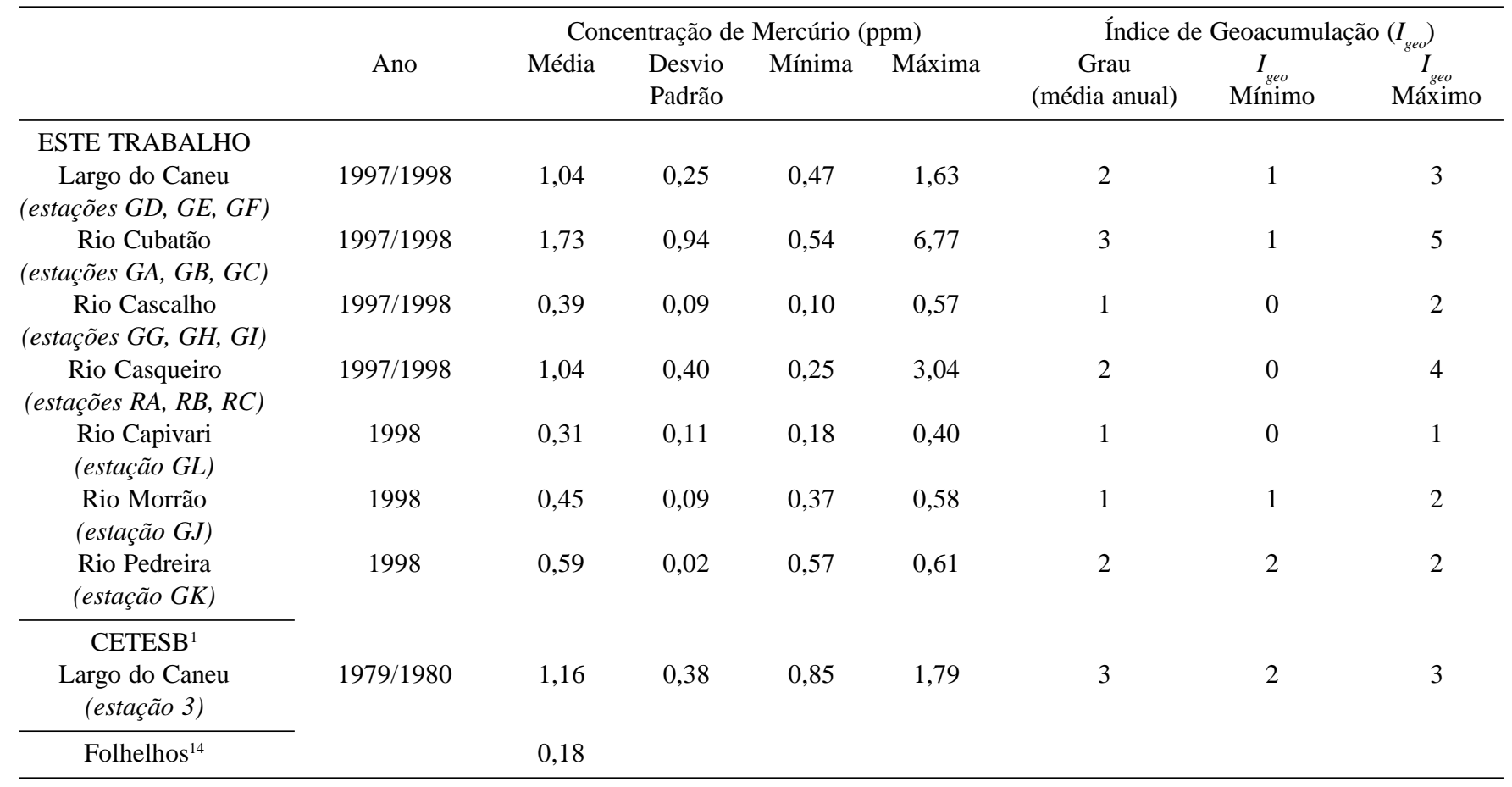




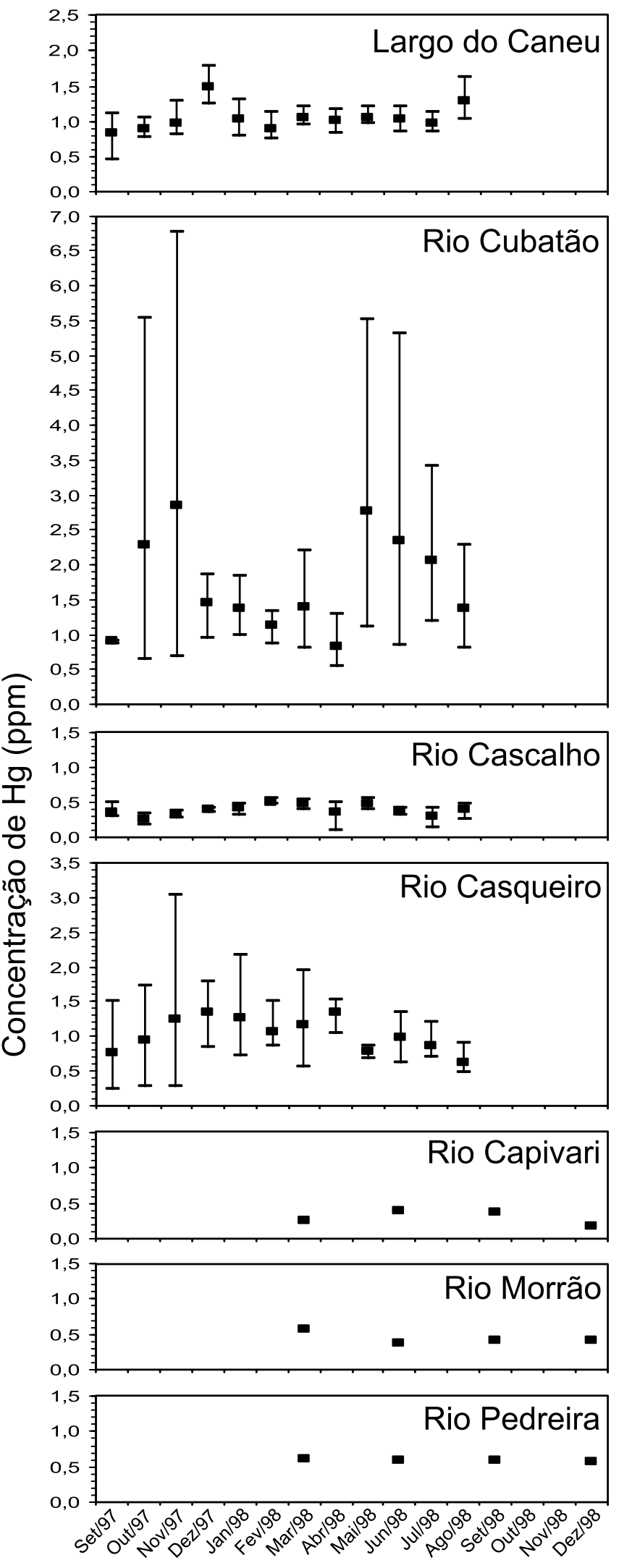

Figura 2. Concentração de Hg em sedimentos superficiais (fração $<63 \mu \mathrm{m}$ ) de alguns rios do Estuário de Santos-Cubatão (SP). Valores médios, máximos e mínimos, relativos a três estações de amostragem, são mostrados no Largo do Caneu e nos rios Cubatão, Cascalho e Casqueiro. Concentrações absolutas são apresentadas nos rios Capivari, Morrão e Pedreira (uma estação de amostragem) te prontamente disponível para os organismos aquáticos, é importante lembrar que algumas espécies de peixes analisadas na área nos anos de 1979 e 1980 apresentaram concentração significativa de $\mathrm{Hg}$ nas vísceras e musculatura ${ }^{3}$. Além disso, se os altos níveis de $\mathrm{Hg}$ nos sedimentos não constituem uma disponibilidade iminente para a biota, a variação de algumas características físicas e químicas $(\mathrm{pH}$, salinidade, potencial redox, etc) das águas circulantes certamente pode promovê-la.

Os dados coletados não permitem afirmar ou refutar que os níveis de $\mathrm{Hg}$ encontrados nos sedimentos do Estuário de SantosCubatão são resultantes de introduções antropogênicas recentes. Isso porque não se pode descartar a presença de sedimentos antigos contaminados nas amostras coletadas. Tal presença pode estar ligada à re-suspensão e amplitude da amostragem (até $3,0 \mathrm{~cm}$ de profundidade), que poderiam incorporar material antigo contaminado. Entretanto, os dados revelam que os sedimentos superficiais deste estuário constituem, por si só, uma fonte potencial de poluição de $\mathrm{Hg}$.

\section{CONCLUSÕES}

O índice de geoacumulação $\left(I_{\text {geo }}\right)$ médio anual de $\mathrm{Hg}$ nos sedimentos superficiais (fração $<63 \mu \mathrm{m}$ ) do Estuário de Santos-Cubatão (1997/1998) ocorre entre os graus 1 e 3, em escala que varia de 0 a 6. O Rio Cubatão apresenta as maiores concentrações deste metal, podendo apresentar, localmente, $I_{\text {geo }}$ de grau 5 , ao se considerar amostras mensais de sedimento. Os valores mais elevados são resultantes de concentração que supera em até 37 vezes os valores de referência (composição média dos folhelhos $=0,18 \mathrm{ppm}^{14}$ ). O Rio Cubatão é, portanto, a principal entrada de $\mathrm{Hg}$ no estuário, rio este que recebe os efluentes de quase todo o setor industrial da cidade de Cubatão.

\section{AGRADECIMENTOS}

R. H. R. Matos agradece ao Conselho Nacional de Desenvolvimento Científico e Tecnológico (CNPq) e G. C. Kristosch, à Coordenação de Aperfeiçoamento de Pessoal de Nível Superior (CAPES) pelo suporte financeiro.

\section{REFERÊNCIAS}

1. CETESB; Metais pesados na Baía de Santos e Estuários de Santos e São Vicente, Relatório, Companhia de Tecnologia de Saneamento Ambiental do Estado de São Paulo: São Paulo, 1981, p. 82.

2. CETESB; Avaliação preliminar da contaminação por metais pesados na água, sedimento e organismos aquáticos do Rio Cubatão (SP), Relatório, Companhia de Tecnologia de Saneamento Ambiental do Estado de São Paulo: São Paulo, 1989, p. 28.

3. Boldrini, C. V.; Pereira, D. N.; Ambiente 1987, 1, 118.

4. Gutberlet, J.; Cubatão: Desenvolvimento, Exclusão Social, Degradação Ambiental, EDUSP: São Paulo, 1996, p. 244.

5. Galvão-Filho, J. P.; Ambiente 1988, 2, 70.

6. Soares, H. M. V. M.; Boaventura, R. A. R.; Machado, A. A. S. C.; da Silva, J. C. G. E.; Environ. Pollut. 1999, 105, 311.

7. Thornton, I.; Applied Environmental Geochemistry, Academic Press: London, 1983, p. 501.

8. Salomons, W.; Förstner, U.; Metals in the Hydrocycle, Springer-Verlag: Berlin, 1984, p. 349.

9. Araújo, M. F. D; Bernard, P. C.; Van Grieken, R. E.; Mar. Pollut. Bull. 1988, $19,269$.

10. Gouveia, M. A.; Araújo, M. F. D.; Dias, J. M. A.; Chem. Geol. 1993, 107, 379 .

11. Mogollón, J. L.; Bifano, C.; Davies, B. E.; Appl. Geochem. 1996, 11, 605.

12. Villaescusa-Celaya, J. A.; Gutiérrez-Galindo, E. A.; Flores-Munõz, G.; Environ. Pollut. 2000, 108, 453.

13. Förstner, U. Em Applied Environmental Geochemistry; Thornton, I., ed.; Academic Press: London, 1983, p. 395.

14. Bowen, H. J. M.; Environmental Chemistry of the Elements, Academic Press: London, 1979, p. 333. 